\title{
Models and Methods in the Meson Theory*
}

\author{
Hideki Yukawa \\ Kyoto University, Kyoto, Japan**
}

\section{INTRODUCTION}

$T$ HE starting point of the meson theory was to express the nuclear forces in terms of the field theory on the basis of Einstein's principle of relativity. The quanta associated with the nuclear field can be regarded, apart from the mass and the charge, as something similar to Einstein's light quanta associated with the electromagnetic field in that both have integer spin and obey Bose-Einstein statistics. The subsequent discovery in cosmic rays of mesons with mass $m_{\mu}$ about two hundred times the electron mass $m_{e}$ lead us naturally to the second assumption that these mesons are identical with the quanta associated with the nuclear field. Moreover, the fact that these mesons are unstable and decay into electrons with the lifetime $\tau_{\mu}$ about $2 \cdot 10^{-6} \mathrm{sec}$. has been considered as a confirmation of the third assumption that the nuclear field interacts with the electron-neutrino system in the same way as with the nucleon, the only difference being a much smaller interaction in order to account for the very slow rate of nuclear $\beta$-decay. The development of the meson theory based on these three fundamental assumptions seemed to be successful in the qualitative interpretation of almost all phenomena so far known in the field of cosmic rays as well as in the field of atomic nuclei. However, it could never arrive at the stage of quantitiative agreement with the experiment.

This rather puzzling situation of the meson theory changed in 1947 due to the experiment by the Rome group on the decay of negative mesons on the one hand, ${ }^{1}$ and the discovery by the Bristol group of two kinds of mesons in cosmic rays on the other. ${ }^{2}$ At first sight these might be looked upon as only adding further confusion and complexity to increasing difficulties in the meson theory. However, it turned out soon that various phenomena connected with cosmic-ray mesons could be understood with less difficulty than ever by accepting the newly established facts that

(i) the mesons mentioned above, which are the main constituent of the hard component of cosmic rays

\footnotetext{
* The main content of this paper was read before the annual meeting of the American Physical Society at Columbia University, New York, on January 28, 1949. The manuscript has been revised and supplemented, however, in order to include results of works which have appeared since then. The author does not claim that this report contains something very original. It is rather a natural outcome of frequent discussions with many physicists, mainly in Princeton and Berkeley. Especially he owes very much to Professor Oppenheimer and Professor Serber.

** At present at the Institute for Advanced Study, Princeton, New Jersey.

${ }_{1}^{1}$ M. Conversi, E. Pancini, and O. Piccioni, Phys. Rev. 71, 209 (1947).

${ }^{2}$ C. M. G. Lattes, H. Muirhead, G. Occhialini, and C. Powell, Nature 159, 694 (1947); C. M. G. Lattes, G. Occhialini, and C. Powell, Nature 160, 453, 486 (1947).
}

observed at sea level, are the lighter ones, i.e., $\mu$-mesons, which interact only very weakly with nucleons, so that they have nothing to do with the nuclear forces, ${ }^{3}$ and that

(ii) the heavier mesons, i.e., $\pi$-mesons with a mass about $300 m_{e}$ interact strongly with nucleons and can decay spontaneously into $\mu$-mesons with a lifetime of the order of $10^{-8} \mathrm{sec}$.

These fundamental facts were further confirmed by experiments concerning mesons artificially produced by the Berkeley cyclotron. ${ }^{4}$

\section{POSSIBLE MODELS}

In order to reformulate a meson thery on the basis of these new facts, it is necessary, first of all, to make a good choice of the spins of $\pi$ - and $\mu$-mesons. We are now well convinced that among four possible combinations

$\begin{array}{lcc}\text { case I } & 0 \text { or } 1 & 0 \\ \text { case II } & 0 \text { or } 1 & \frac{1}{2} \\ \text { case III } & \frac{1}{2} & 0 \\ \text { case IV } & \frac{1}{2} & \frac{1}{2}\end{array}$

taken up by Marshak and Bethe, ${ }^{5,6}$ case II is very likely the one realized in nature. Arguments in favor of choosing case II were made by Oppenheimer, Serber, Wheeler, and others on various occasions. Similar arguments were also made in Japan, especially by Taketani. ${ }^{7}$ The most decisive points of these arguments are the striking contrast in the results of capture of $\pi$ and $\mu$-mesons by nuclei. When a slow negative $\pi$-meson is captured by a nucleus, most of its rest energy $m_{\pi} c^{2}$ $=150 \mathrm{Mev}$ is transferred as kinetic energy to nuclear particles accompanying the disruption of the whole nucleus. This is possible only if $\pi$-mesons have integer spin and no neutral particles other than neutrons are

${ }^{3}$ The latest value for the mass of $\mu$-mesons in cosmic rays is $212 m_{e} \pm 5 m_{e}$ according to R. B. Brode, Phys. Rev. 75, 904 (1949). The most accurate value for the lifetime $\tau_{\mu}$ of $\mu$-mesons is probably $2.15 \cdot 10^{-6}$ sec. obtained by N. Nereson and B. Rossi, Phys. Rev. 64, 199 (1943).

4 E. Gardner and C. M. G. Lattes, Science 107, 270 (1948) ; W. Barkas, E. Gardner, and C. M. G. Lattes, Phys. Rev. 74, 1558 (1948); J. R. Richardson, Phys. Rev. 74, 1720 (1948). Latest values of the mass and the lifetime of $\pi$-mesons artificially produced in Berkeley are $m_{\pi}=285 m_{e}$ and $\tau_{\pi}=1.11 \cdot 10^{-8} \mathrm{sec}$., respectively. The mass of $\mu$-mesons is $m_{\mu}=216 m_{e}$ in good agreement with the value for cosmic-ray mesons obtained by Brode.

5 R. E. Marshak and H. A. Bethe, Phys. Rev. 72, 506 (1947).

${ }^{6}$ Spin 1 for $\mu$-mesons is ruled out by considerations on burst production according to R. F. Christy and S. Kusaka, Phys. Rev. 59, 414 (1941).

${ }_{7}$ M. Taketani, S. Nakamura, K. Ono, and M. Sasaki, Kagaku 18, 564 (1948); M. Taketani, S. Nakamura, K. Ono, and M. Sasaki, Phys. Rev. 76, 60 (1949). See also H. Yukawa, Prog. Theor. Phys. 3, 217A (1948), 
emitted simultaneously, as discussed in detail by Wheeler and Tiomo. ${ }^{8}$ The possibility of assigning spin $\frac{1}{2}$ to $\pi$-mesons is excluded because a meson with spin $\frac{1}{2}$ can never be captured by a nucleus without emitting a neutrino or a neutral meson with spin $\frac{1}{2}$, which takes away most of the rest energy of the incident $\pi$-meson. On the contrary, $\mu$-mesons give rise very seldom, if any, to nuclear disruption of the above type, which in turn implies to assign spin $\frac{1}{2}$ to $\mu$-mesons. ${ }^{9}$

Now, if we accept these fundamental assumptions corresponding to case II, the interpretation of elementary processes connected with the creation or annihilation of mesons follows without serious difficulty: $:^{10}$

(i) $\pi$ - $\mu$-decay: $:^{11} \pi(0,1) \rightarrow \mu\left(\frac{1}{2}\right)+\nu\left(\frac{1}{2}\right)$

(ii) $\mu$ - $\beta$-decay: $:^{12} \mu\left(\frac{1}{2}\right) \rightarrow e\left(\frac{1}{2}\right)+2 \nu\left(\frac{1}{2}\right)$

(iii) slow $\pi$-nuclear-capture $:^{13} \pi^{-}(0,1)+\left\{P\left(\frac{1}{2}\right)+\cdots\right\}$

(iv) slow $\mu$-nuclear-capture : $:^{14} \mu^{-}\left(\frac{1}{2}\right)+\left\{P\left(\frac{1}{2}\right)+\cdots\right\}$ $\rightarrow N\left(\frac{1}{2}\right)+\cdots$ $\rightarrow \nu\left(\frac{1}{2}\right)+N\left(\frac{1}{2}\right)+\{\cdots\}$.

The simplest modal corresponding to case II in conformity with the general idea of the meson theory is just the one, which was proposed by Sakata ${ }^{15}$ in 1943 before the discovery of $\pi$ - and $\mu$-mesons in cosmic rays. This, in turn, is a modification of a modal proposed by Tanikawa, ${ }^{16}$ which corresponds to case I. The aim of their attempts at that time was to remove the wellknown discrepancy between theoretical and observed lifetimes of cosmic-ray mesons on the one hand and that between theoretical and observed cross sections of scattering of these mesons by nucleons by modifying the second assumption of the existing meson theory. Thus, they assumed the existence of two kinds of mesons, the heavier one being responsible for the

${ }^{8}$ J. A. Wheeler and J. Tiomno, Phys. Rev. 75, 1306 (1949); Rev. Mod. Phys. 21, 153 (1949). See also R. Serber, Phys. Rev. 75, 1459A (1949).

${ }_{9}$ An alternative assumption of assigning spin 0 to $\mu$-mesons, which are coupled to nucleons only in pairs, is very unlikely for several reasons.

${ }^{10}$ In the following, $\pi, \mu, \nu, e, P$, and $N$ denote $\pi$-meson, $\mu$-meson, neutrino, electron, proton, and neutron, respectively. The number in brackets indicates the spin of the particle. The suffix \pm or 0 indicates whether the particles are electrically positive, negative or neutral. $\{\cdots\}$ means that the particles are bound together

${ }^{11}$ Recent observations by E. Gardner, C. M. G. Lattes, and A. Bishop, Phys. Rev. 75, 1468A (1949) are in good accord with this assumption, although the possibility $\pi(0,1) \rightarrow \mu\left(\frac{1}{2}\right)+\mu^{0}\left(\frac{1}{2}\right)$ cannot be ruled out, provided that the neutral $\mu$-meson $\mu^{0}$ has a mass of the order of $m_{e}$.

${ }_{12}$ For example, recent results obtained by C. Anderson, R. Leighton, and A. Seriff, Phys. Rev. 75, 1466A (1949), are strongly in favor of the above mechanism. An alternative process $\mu\left(\frac{1}{2}\right) \rightarrow e\left(\frac{1}{2}\right)+\nu\left(\frac{1}{2}\right)+\mu^{0}\left(\frac{1}{2}\right)$ cannot be excluded for the time being.

${ }_{13}$ The probability of other processes such as $\pi^{-}(0,1)+P\left(\frac{1}{2}\right) \rightarrow \gamma$ due to the weakness of coupling of the electromagnetic field with the meson and the nucleon compared with that between the meson and the nucleon.

${ }^{14}$ For example, recent experiments by W. Y. Chang, Rev. Mod. Phys. 21, 166 (1949) can only be interpreted very well by the above mechanism. Again an alternative process $\mu^{-}\left(\frac{1}{2}\right)+\cdots \rightarrow$ $\mu^{0}\left(\frac{1}{2}\right)+\cdots$ cannot be ruled out.

${ }^{15} \mathrm{~S}$. Sakata and T. Inoue, Prog. Theor. Phys. 1, 143 (1946).

${ }^{16}$ Y. Tanikawa, Prog. Theor. Phys. 2, 220 (1947). nuclear forces as well as $\beta$-decay and the lighter one constituting the main part of the hard component of cosmic rays at sea level. Obviously, they can now be identified with the $\pi$-meson and the $\mu$-meson, respectively.

Thus we arrive at a simple model (Fig. 1), but we have to modify it further for following reasons. First of all, the lifetime of $\mu$-mesons with respect to $\mu$ - $\beta$-decay is obviously far too long compared with the observed value $2 \cdot 10^{-6}$ sec., because the probability of $\mu$ - $\beta$-decay is proportional to the product of the squares of two small coupling constants $g^{\prime}$ and $g^{\prime \prime} .{ }^{17}$ Another difficulty is that, if we want to interpret nuclear $\beta$-decay as a consequence of virtual creation and annihilation of $\pi$-mesons, the coupling constant $g^{\prime}$ becomes so large that the lifetime of $\pi$-mesons with respect to $\pi$ - $\beta$-decay is even shorter than the observed lifetime with respect to $\pi$ - $\mu$-decay..$^{18}$ This is certainly in contradiction with the fact that any competing process must have much longer lifetime compared with $\pi$ - $\mu$-decay. ${ }^{19}$

Thus we have to replace the third assumption of the meson theory with another one. Apparently there are several possible ways of modifying Fig. 1 . The first one is to assume a direct coupling between the nucleon and the electron-neutrino and that between the $\mu$-mesonneutrino and the electron-neutrino instead of the coupling between the $\pi$-meson and the electron-neutrino (Fig. 2). An advantage of this model is that three processes, i.e., $\pi$ - $\mu$-decay, $\pi$-nuclear capture, and $\mu$ nuclear capture, can be consistently accounted for by adjusting two constants $g$ and $g^{\prime \prime}, 20$ just as in the case of Fig. 1. Incidentally we find that the other two constants $g_{F}$ and $g_{\mu e}$ are of the same order of magnitude.

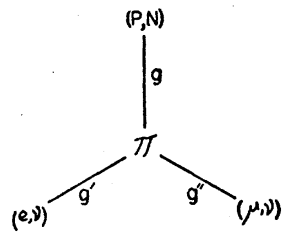

Fig. 1. Model I.

${ }^{17} g^{\prime}$ must be small in order to account for the nuclear- $\beta$-decay, and $g^{\prime \prime}$ must be also small in order to account for the observed lifetime of $\pi$ - $\mu$-decay.

${ }^{18}$ L. I. Schiff, Phys. Rev. 74, 1556 (1948). Note added in proof: However, there is a peculiarity in the case of the pseudoscalar $\pi$-meson. Namely, the ratio of the probabilities of $\pi$ - $\mu$-decay and $\pi$ - $\beta$-decay is roughly equal to $m_{\mu}^{2} / m_{e}^{2} \sim 4 \times 10^{4}$, if we assume the same pseudovector coupling for $\pi-\mu$ - and $\pi$-electron, as can be inferred from previous calculations by S. Sakata, Proc. Phys.Math. Soc. Japan 23, 291 (1941). Thus, if we take advantage of the pseudoscalar $\pi$-meson, the direct interaction between the nucleon and the electron-neutrino as well as that between the nucleon and the $\mu$-meson-neutrino may not be necessary, although the direct interaction between the $\mu$-meson-neutrino and electronneutrino is still indispensable, unless we introduce more mesons with the integer spin.

${ }_{19}$ C. M. G. Lattes, Phys. Rev. 75, 1468A (1949).

${ }^{20}$ A. Lodge, Nature 161, 809 (1948); C. Marty and J. Prentki, J. phys. et rad. 9, 147 (1948); T. Inoue and S. Ogawa, Prog. Theor. Phys. 3, 319 (1948); J. L. Lopes, Phys. Rev. 74, 1722 (1948); Bruno, Arkiv. f. Mat. Astr. o. Fys. 36, No. 8 (1948); Arkiv. f. Fys. 1, No. 2 (1949); R. Latter and R. F. Christy, Phys. Rev. 75, 1459A (1949). 

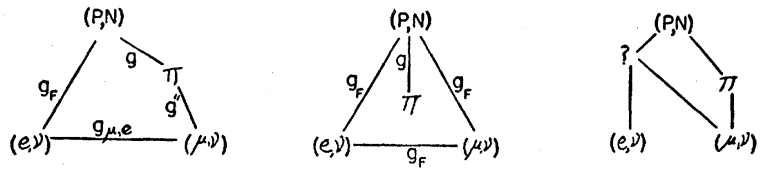

FIG. 2. Model II.

FIG. 4. Model IV.

The second alternative is to assume all three kinds of direct coupling between any two of three kinds of spinor particles in place of the coupling between the $\pi$-meson and the $\mu$-meson-neutrino and that between the former and the electron-neutrino, as proposed by Tiomno and Wheeler ${ }^{21}$ (Fig. 3). There is a remarkable simplicity in this model due to the circumstance that three constants of direct coupling are of the same order of magnitude, and indeed, it is conceivable that there is only one common Fermi's constant $g_{F}$ of the order of $10^{-49} \mathrm{erg} \mathrm{cm}^{3}$. Thus, the interaction of elementary elementary particles can be divided into three groups characterized by three coupling constanta $g, e$, and $g_{F}$, respectively. ${ }^{22}$ However, this advantage is partly offset by the difficulty that the probability of $\pi$ - $\mu$-decay as well as that of $\pi$ - $\beta$-decay due to virtual creation and annihilation of nucleon pairs diverge $\mathrm{e}^{23}$ or, if they are made finite by some prescription, they will have in general the same order of magnitude contrary to experiment. ${ }^{19}$

Furthermore, there is another sort of difficulty common to model II and model III (Figs. 2 and 3). Namely, the introduction of a direct coupling between spinor particles is always accompanied by the appearance of singularities proportional to the inverse fifth power of the distance between two similar spinor particles as was familiar from pair theories of nuclear forces. Although this may well be a problem which can only be solved by future developments of field theory, alternatively, one can get rid of this difficulty by postulating the existence of other mesons in addition to the $\pi$-meson, which have integer spin and are coupled with three kinds of Fermi particles (Fig. 4). In this case, however, things become so arbitrary that we can hardly prefer one model to all others. ${ }^{24}$

${ }^{21} \mathrm{~J}$. Tiomno and J. A. Wheeler, Rev. Mod. Phys. 21, 144, 153 (1949). See also Lee, Rosenbluth, and Yang, Phys. Rev. 75, 904 (1949). The author is indebted to Professor Wheeler and Mr. Tiomno for informing him of their calculations before publication.

${ }^{22}$ It is a matter of course that we have to add the interaction of photons with protons, electrons and charged $\pi$ - and $\mu$-mesons characterized by the common constant $e$ to each of the models. The simplicity of Fig. 3 mentioned above gives rise to new questions as to possible underlying meaning. See, for example, Klein, Nature 161, 897 (1948).

${ }^{23}$ R. Marshak, Phys. Rev. 75, 700 (1949). The divergence is the same as in the case of S. Sakata's calculation of meson decay (Phys. Rev. 58, 576 (1940); Proc. Phys.-Math. Soc. Japan 23, 283 (1941)).

${ }^{24}$ For example, M. Sasaki, S. Nakamura, and S. Hayakawa (Prog. Theor. Phys. 3, $454(1948)$ ) assume the existence of another meson with integer spin and a mass of the order of $200 m_{e}$, which is. strongly coupled with the nucleon and decays into an electron and a neutrino with a lifetime of the order of $10^{-8}$ sec. or shorter. Thus they tried to account for the deuteron quadrupole moment and the $\beta$-decay. They proposed further to identify this meson

\section{PROBLEMS OF THE $\tau$-MESON AND THE NEUTRAL MESON}

In order to complete any one of the models, one has to take into account another new fact of great importance. Indications for the existence of mesons with a mass of the order of $1000 m_{e}$ were reported more than once, ${ }^{25}$ and recent observation by Leprince-Ringure and others seems to show the occurrence of nucleat disruption caused by a meson with a mass about $1000 m_{e}{ }^{26} \mathrm{~A}$ photograph, obtained by Powell and others very recently, ${ }^{27}$ clearly shows a remarkable phenomenon of decay of the so-called $\tau$-meson with a mass about $1000 m_{e}$ into three mesons, one of which is very probably a negative $\pi$-meson. These new discoveries open the way to various possibilities, but at the same time give rise to serious difficulties. On the one hand, the fact that the $\tau$-meson can originate a nuclear disruption similar to that originated by the $\pi$-meson seems to be in favor of the theory of mixed meson fields first proposed by $\mathbf{M} \phi 1$ ler and Rosenfeld ${ }^{28}$ and later extended by Schwinger ${ }^{29}$ or any modification, which assumes the coexistence of mesons with integer spin and different masses, both strongly coupled with nucleons. ${ }^{30}$ On the other hand, however, such an assumption leads in general to a serious difficulty as to the stability of heavier mesons, because they must decay into lighter mesons and photons in a very short time. According to Finkelstein, ${ }^{31}$ the lifetime of a charged vector meson with respect to the decay into a charged pseudoscalar meson and a photon may be as short as the order of $10^{-18} \mathrm{sec}$. This process is intimately connected with well-known decay of the neutral meson with integer spin and strongly coupled with the nucleon into two or three photons as first calculated by Sakata and Tanikawa $^{31,32}$ The latter processes give very short lifetimes of the order of $10^{-16} \sim 10^{-11}$ sec. to the pseudoscalar and vector mesons. ${ }^{33}$ For $\tau$-mesons, which have

with so-called $N$-radiation in cosmic rays. See also Y. Tanikawa, Prog. Theor. Phys. 3, 314 (1948).

${ }_{25}^{25}$ L. Leprince-Ringuet and M. L'Heritier, Comptes Rendus 219, 618 (1944); J. de phys. et rad. 7, 66 (1946); G. D. Rochester and C. C. Butler, Nature 20, 855 (1947)

${ }^{26}$ Leprince-Ringuet, Fong, Janneau, and Morellet, Comptes Rendus 226, 1897 (1948); L. Leprince-Ringuet, Rev. Mod. Phys. 21, 42 (1949).

${ }^{27}$ Brown, Camerini, Fowler, Muirhead, Powell, and Ritson, Nature 163, 47, 82 (1949).

${ }^{28} \mathrm{C}$. M $\phi$ ler and L. Rosenfeld, Kgl. Danske Vid. Sels. Math.fys. Medd. 17, No. 8 (1940).

${ }^{29} \mathrm{~J}$. Schwinger, Phys. Rev. 61, 387 (1942).

${ }^{30}$ Various possible combinations of meson fields were investigated by many authors. However, we have to reconsider the whole problem from the new standpoint, which will be mentioned later.

${ }_{31}$ R. J. Finkelstein, Phys. Rev. 72, 415 (1947).

${ }^{32}$ S. Sakata and Y. Tanikawa, Phys. Rev. 57, 548 (1940).

${ }^{33}$ However, according to recent calculations by Miyamoto and Fukuda to be published in Prog. Theor. Phys. 4 (1949), the lifetime depends very much on whether the terms, which are not gauge-invariant, are retained or not. If such terms are omitted, the lifetime becomes of the order of $10^{-9} \sim 10^{-8}$ sec. Note added in proof: Extensive calculations of the probabilities of various decay processes were made by Steinberger by using Pauli's regulator. See also S. Sasaki, S. Oneda and S. Ozaki, Prog. Theor. Phys. 4 (1949), in press. 
a mass much larger than that of $\pi$-mesons, there are two or more possible modes of decay. These are, in addition to the above-mentioned decay into a $\pi$-meson and a photon due to the process through intermediate states such as

$$
\tau^{+} \rightarrow(N)+P \rightarrow(N)+P^{\prime}+\gamma \rightarrow \pi^{+}+\gamma,
$$

where ( ) means an antiparticle, a $\tau$-meson can also decay into a charged $\pi$-meson and a neutral $\pi$-meson, provided that the latter exists and has a mass nearly equal to $m_{\pi}$, due to the process through intermediate states such as

$$
\tau^{+} \rightarrow(N)+P \rightarrow(N)+N^{\prime}+\pi^{+} \rightarrow \pi^{0}+\pi^{+} .
$$

Furthermore, if the mass of the $\tau$-meson $m_{\tau}$ is larger than $3 m_{\pi}$, it can decay into three $\pi$-mesons through intermediate states such as

$$
\begin{aligned}
\tau^{+} & \rightarrow(N)+P \rightarrow(N)+N^{\prime}+\pi_{1}^{+} \\
& \rightarrow(N)+P^{\prime}+\pi_{2}^{-}+\pi_{1}^{+} \rightarrow \pi_{3}^{+}+\pi_{2}^{-}+\pi_{1}^{+} .
\end{aligned}
$$

According to a preliminary result of calculation by Nakamura, ${ }^{34}$ the lifetime of the $\tau$-meson (with spin 1) due to the decay into three $\pi$-mesons (with spin 1 ) is of the order of $10^{-11} \mathrm{sec}$. or longer, as can be expected from the above experimental informations, if we assume $g_{\tau}{ }^{2} / \hbar c$ to be of the order of $10^{-4}$ or smaller, where $g_{\tau}$ is the coupling constant between the $\tau$-meson and the nucleon. ${ }^{35}$ Thus, this process may possibly be identified with the $\tau$-three-meson-decay observed by Powell and others, although the possibility of identifying two of the three mesons with $\mu$-mesons instead of $\pi$-mesons cannot be excluded..$^{36}$ However, the theoretical lifetime of the $\tau$-meson due to any other competing process such as (1) or (2) is in general much shorter than $10^{-11} \mathrm{sec}$., so that the length of the slow $\tau$-meson track becomes too short to be observed in contradiction with the experiment. ${ }^{37}$ Moreover, in contrast to the case of $\gamma$-decay of the neutral meson, there seems to be no selection rule based on Furry's theorem, ${ }^{38}$ because the probability amplitude relevant to the decay of the charged meson is no longer symmetric with respect to

\footnotetext{
${ }^{34} \mathrm{~S}$. Nakamura, private communication.

${ }_{35}$ If $g_{\tau}$ is too small, one cannot understand how $\tau$-mesons are produced in the atmosphere.

${ }^{36}$ According to Pais and Power, the lifetime of $\tau$-meson due to the decay into a $\pi$-meson and two $\mu$-mesons turns out to be of the order of $10^{-9} \mathrm{sec}$., if we assume a direct coupling between the $\mu$-meson pair and the nucleon with the same coupling constant as Fermi's constant $g_{F}$. The trouble is that the sign of the charge of the $\tau$-meson and that of the $\pi$-meson have to be the same in contradiction to Powell's observation, which seems to imply a positive $\tau$-meson and a negative $\pi$-meson. The author is indebted to them for informing him of this result before publication.

${ }^{37}$ In fact, van Wÿck calculated the probability of the process of the type (1) for various types of meson fields and found that the lifetime of the $\tau$-meson was always too short to account for the experiment, unless we take $g_{\tau}{ }^{2} / \hbar c$ as small as $10^{-7} \sim 10^{-5}$ corresponding to the lifetime $10^{-9} \sim 10^{-11} \mathrm{sec}$. The author is indebted to Professor Oppenheimer and Professor Peierls for giving him the opportunity of reading van Wÿck's paper before publication.

${ }^{38}$ W. H. Furry, Phys. Rev. 51, 125 (1937).
}

the exchange of the nucleon and the anti-nucleon. ${ }^{39} \mathrm{~A}$ possible way of getting rid of this difficulty in the framework of the present meson theory may be found, if the peculiarity similar to that, which was claimed by Miyamoto and Fukuda in the case of two- $\gamma$-decay of the neutral meson, ${ }^{33}$ turns out to appear also in other cases so as to prolong the lifetime sufficiently. Otherwise, we have to expect some fundamental alteration in relativistic properties of the nucleon, because it is hard to do without neutral mesons as will be discussed in the following.

\section{PROBLEMS OF NUCLEAR FORCES}

Now we have to consider the present situation of the problem of the nuclear forces, which is undoubtedly the central problem of meson theory. ${ }^{40}$ As is very well known, among 12 cases, which can be obtained by considering neutral, charged and symmetric theories for each of four types of meson fields, i.e., scalar, vector, pseudoscalar, and pseudovector fields, the symmetric pseudoscalar theory is probably the best one, in that it is by itself in agreement with the experiment, at least qualitatively. ${ }^{41}$ However, even this case has been considered to be unsatisfactory, mainly because the potential between two nucleons obtained by straightforward perturbation calculation to the second order contained an inadmissible singularity proportional to $r^{-3}, r$ being the distance between two nucleons. Singularities of the same type appeared also in the cases of vector and pseudovector fields. Then the mixed field theories came out in order to remove these singularities by compensation. However, it turned out recently that two types of coupling between the pseudoscalar meson and the nucleon, i.e., the pseudoscalar and pseudovector couplings, were equivalent to each other as pointed out by Dyson ${ }^{42}$ and proved rigorously by Case by applying Schwinger-Tomonaga's method. ${ }^{43}$ Thus, as shown by van Hove, ${ }^{44}$ it became clear that the nuclear potential contained no singularity higher than $r^{-1}$, if the secondorder calculation was performed relativistically with respect to the nucleons, whereas the potential due to the vector field still contains a singularity proportional to $r^{-3}$. Under these circumstances, it seems better to consider the pseudoscalar meson field alone instead of mixing the vector or pseudovector meson field together.

${ }^{39}$ However, there are other kinds of selection rules associated with the conservation of the angular momentum, parity, etc. as pointed out by Peierls and van Wyck. In this connection, Power arrived at an interesting conclusion that the lifetime of $\tau$-mesons can be long enough to be observed, only if we assume that $\tau-\pi$ and neutral mesons are all pseudoscalar.

40 A detailed account of the whole subject is found in L. Rosenfeld, Nuclear Forces I (1948); Nuclear Forces II (1949), NorthHolland Publ. Co. (Amsterdam); Interscience Publishers (New York).

${ }_{41}$ W. Rarita and J. Schwinger, Phys. Rev. 59, 436, 556 (1941);

R. Oppenheimer, Phys. Rev. 59, 462 (1941).

${ }^{42}$ F. J. Dyson, Phys. Rev. 73, 929 (1948).

${ }^{43}$ K. M. Case, Phys. Rev. 75, 1306 (1949).

${ }^{44}$ L. Van Hove, Phys. Rev. 75, 1519 (1949). See also H. Goldstein and H. Feshbach, Phys. Rev. 75, 1306 (1949). Similar results were also obtained by Nambu, Prog. Theor. Phys. 3, 444 (1948). 
Now, for small values of $r$ comparable with $\hbar / M c$, where the problem of divergence is serious, the accuracy of the second-order calculation is poor, because the effect of higher order terms cannot be neglected. On the contrary, for large values the nuclear potentials obtained by relativistic second-order calculations should be the same as the well known formulas obtained by non-relativistic calculations..$^{45}$ Thus, although we are convinced now that the mixed theory is of no use in removing the divergence, we are not yet sure whether it is necessary to introduce two or more meson fields for other reasons. At any rate, the new situation is very much in favor of the pseudoscalar meson theory. Moreover, as shown by Case, ${ }^{46}$ the anomalous magnetic moments of the neutron and the proton become finite and are of the correct order of magnitude, if we apply Schwinger-Tomonaga's method to the pseudoscalar meson theory, in contrast to previous divergent results obtained by usual perturbation theory. ${ }^{47}$ The neutronelectron interaction, which was discovered recently by Rabi and others, ${ }^{48}$ can be calculated in a similar way. ${ }^{46,49}$ The potential between the neutron and the electron thus obtained has a depth of the order of a few kev extending over a distance of the order of the electron radius, as expected from experiment. However, it is impossible to obtain quantitative agreement with experiment both for the magnetic moment and the neutron-electron interaction. The reason for the discrepancy is still very obscure..$^{50}$

In this connection, it is to be noticed that the pseudoscalar coupling and the pseudovector coupling are equivalent to each other, if the corresponding coupling constants $f$ and $g$ satisfy the relation $f=2 M / \mu \cdot g$, where $\mu$ is the mass of the pseudoscalar meson. Thus, if we assume the pseudoscalar coupling alone, $f^{2} / \hbar c$ must be much larger than $g^{2} / \hbar c$, which is of the order of $10^{-1}$. Then, for small values of the distance $r$ between two nucleons, fourth- and higher order effects become very large and we have to deal with strong coupling.

\footnotetext{
${ }^{45}$ In this connection, G. Araki (Phys. Rev. 75, 1262 (1949); Prog. Theor. Phys. 4 (1949), in press) derived recently the nuclear potentials by using a method of calculation, which takes into account only the contribution from the non-relativistic region for the nucleons. These potentials are equivalent to the conventional potentials for larger $r$ as it should be, but are different from those which were obtained by relativistic calculations for smaller $r$ in the cases of vector and pseudovector fields, in that Araki's potentials contain no singularity higher than $r^{-1}$ even in these cases. These potentials may be of some practical use, although they cannot claim to have fundamental significance.

${ }_{46}$ K. M. Case, Phys. Rev. 74, 1884 (1948); 76, 1 (1949).

${ }^{47}$ However, J. M. Luttinger (Helv. Phys. Acta 21, 483 (1948); Phys. Rev. 75, 309 (1949)) derived the same result as Case by using a method nearer to the ordinary perturbation theory.

${ }^{48}$ Havens, Rabi, and Rainwater, Phys. Rev. 72, 634 (1947);

Rainwater, Rabi, and Havens, Phys. Rev. 75, 1295 (1949).

${ }_{49}$ M. Slotnick and W. Heitler, Phys. Rev. 75, 1645 (1949).

50 In the cases of vector and pseudovector fields with the tensor coupling, the divergence difficulties still remain after performing mass and charge renormalizations according to Schwinger-Tomonaga's method as shown by Ning Hu, Phys. Rev. 75,1305 (1949), so that the comparison of different meson models is very difficult for the time being.
}

Now, the theory of strong coupling originated by Wentzel ${ }^{51}$ has been developed and applied to various types of meson field. ${ }^{52,53}$ Although these investigations contributed to reveal some new features of nuclear forces, which are essentially different from those features well-known in perturbation theory, the strong coupling theory has to suffer always from the serious restriction that a finite size must be given to the nucleon in order to avoid the divergence of the whole calculation. This corresponds to the use of some more or less conventional cut-off procedure, which destroys the invariance and uniqueness of results thus obtained. Moreover, the fact that the additional magnetic moment of the proton is nearly the same in absolute magnitude with the magnetic moment of the neutron indicates that the usual weak coupling theory is the better approximation to most problems in the low energy region compared with the strong coupling theory, because the latter gives the result that the total magnetic moment of the proton must be nearly equal to the absolute value of that of the neutron contrary to the experiment. On the other hand, it is quite certain that for small distances between the nucleons or, in other words, for high energy collision processes, the effect of strong coupling is very important, but this is just the region, where relativistic properties of the nucleons may play essential roles. It is still an open question to what extent the relativistic formulation of field theory, which has been so successful in quantum electrodynamics, will be able to dispose of these difficulties in the meson theory.

Whatever be the ultimate solution of the problem, one can expect that the large contributions of fourthand higher order terms to nuclear forces may give a sufficient amount of ordinary forces, even if we start from the pure charged pseudoscalar meson theory with pseudoscalar coupling. ${ }^{54}$ However, the range of ordinary forces thus obtained will be much smaller than that of the exchange forces obtained by second-order calculations. On the other hand, according to the well-known result by Breit and others, ${ }^{55}$ proton-proton scattering experiments up to the energy of a few Mev can best be accounted for by assuming the range of force between two protons equal to the range which correspond to the neutral meson with the mass nearly equal to that of the charged $\pi$-meson. Thus, the neutral meson seems to be still indispensable in accounting for the nuclear forces,

51 G. Wentzel, Helv. Phys. Acta 13, 269 (1940); 14, 633 (1941). ${ }^{52}$ As to the details of the strong coupling theory, see summary reports by G. Wentzel, Rev. Mod. Phys. 19, 1 (1947) and by W. Pauli, Meson Theory of Nuclear Forces (Interscience Publishers, Inc., New York, 1946).

${ }^{53} \mathrm{~A}$ method was developed by S. Tomonaga (Prog. Theor. Phys. 1, 83, 109 (1946) ; 2, 6, 63 (1947), which could be applied to the case of intermediate coupling, too.

${ }^{54}$ This possibility was considered by F. J. Dyson in connection with the application of Schwinger-Tomonaga's method to meson theory and worked out by Bethe, Lepore, and Watson. However, their results are not encouraging.

${ }_{55}$ Breit, Hoisington, and Share, Phys. Rev. 56, 884 (1939). See also recent calculations by C. L. Critchfield and D. C. Dodder, Phys. Rev. 75, 419 (1949). 
although the direct evidence for their existence is scarce. ${ }^{56}$

In this connection, high energy neutron-proton scattering experiments in Berkeley ${ }^{57}$ give us very important information. The fact that neutrons with energies as high as $90 \mathrm{Mev}$ are scattered by protons with about equal probability in forward and backward directions (to be more precise, with the probability a little larger in backward direction than in forward direction) clearly shows that the interaction between neutrons and protons is a mixture of about equal amount of exchange and ordinary forces (probably the former being a little larger than the latter), because the pure exchange force gives a predominantly backward scattering and the pure ordinary force gives a predominantly forward scattering at such a high energy region. ${ }^{58}$ Thus, among three typical cases considered hitherto in meson theory, the symmetric theory is certainly much better than the pure charged and pure neutral theories. However, calculations by many authors show that the symmetric theory, too, cannot reach complete agreement with experiment. ${ }^{59}$ As first pointed out by Serber, ${ }^{60}$ a combination of exchange and ordinary forces of relative magnitude about $1: 1$ seems to fit better with the experiment than the symmetric theory, in which the relative contribution is $2: 1$. Anyhow it is still an open question whether there is a simple model which is able to account for high energy scattering adequately, as things are very complicated due to the presence of tensor forces and relativistic effects. As pointed out by Snyder and Marshak, ${ }^{61}$ calculations using the same potential, which are valid for the low energy region, may not be accurate enough to be compared with experiments in the high energy region.

In the region of still higher energy, mesons can be created by collision of two nucleons as was remarkably demonstrated by experiments in Berkeley. The origin of mesons in cosmic rays has long been ascribed to the same type of processes in much higher energy regions. Obviously the most important question in connection

${ }^{56}$ According to H. York, B. Moyer, and R. Bjorklund (Phys. Rev. 76, 187 (1949)), high energy $\gamma$-rays produced by collision of $350 \mathrm{Mev}$ protons with Be nuclei can be accounted for consistently by assuming the initial production of neutral mesons with the mass somewhat larger than the charged $\pi$-meson, which decay into two photons in a very short time. This is again in conformity with the assumption that the neutral $\pi$-meson is pseudoscalar.

${ }^{57}$ Hadley, Kelly, Leith, Segrè, Wiegand, and York, Phys. Rev. 73, 1114 (1948); 75, 351 (1949); Brueckner, Hartsough, Hayward, and Powell, Phys. Rev. 75, 555 (1949). See also Cook, McMillan, Peterson, and Sewell, Phys. Rev. 72, 1264 (1947); 75, 7 (1949).

58 For $90-\mathrm{Mev}$ neutrons, the de Broglie wave-length divided by $2 \pi$ is $0.95 \cdot 10^{-13} \mathrm{~cm}$, which is a little smaller than the range of the nuclear force usually adopted.

${ }_{59}$ As there are so many papers on this subject, it is impossible to cite all of them here. Most of the results published in 1948 can be found in L. Rosenfeld, Nuclear Forces II, 450 (1949). See further F. Rohrlich and J. Eisenstein, Phys. Rev. 75, 705 (1949); R. Christian and E. Hart, Phys. Rev. 75, 1465A (1949); T. Y. Wu and H. M. Foley, Phys. Rev. 75, 1681 (1949).

${ }^{60}$ The author is indebted to Professor Serber for giving him theoretical and experimental information in Berkeley.

${ }^{61}$ H. Snyder and R, E. Marshak, Phys. Rev. 72, 1253 (1947). with the meson theory is whether the same coupling between the $\pi$-meson and the nucleon is effective both for the nuclear forces in low energy region and the creation of $\pi$-mesons by nuclear collision in high energy region. Extensive theoretical investigations were made by Heitler and others, ${ }^{62}$ based on their theory of radiation damping and on M $\phi$ ller-Rosenfeld's model. Their results are as good as we can expect under the situation, which prevents us from choosing one model as superior to all others. However, there are still many questions open to further discussions. Above all, the explosion-like production of mesons by extremely high energy nuclear collision can only be accounted for by considering the strong coupling between colliding nucleons for very small distance as done by Oppenheimer and others. ${ }^{63}$ They concluded that meson emission was multiple and the multiplicity increased with energy for strong coupling as in the case of the pseudoscalar theory, whereas this was not true for weaker coupling as in the case of the scalar theory. In the region of moderately high energy, where only one meson can be created at a time, agreement between theory and experiment seems to be fairly good as far as the nuclear forces are dealt with more or less phenomenologically. ${ }^{64}$ Comparison of pure field theoretical calculations and experiments for the simplest processes such as the creation of $\pi$-mesons by neutron-proton, protonproton, and photon-proton collisions in the near future will probably decide the type of the $\pi$-meson field and its coupling with the nucleon.

Thus, the situation of the meson theory on the whole is not so bad as it was a few years ago. However, we are still at a stage of accepting different kinds of mesons, which have been discovered one after another, only as such. We know nothing about the fundamental law determining the masses of various kinds of elementary particles, which seem to us to be so irregularly distributed. ${ }^{65}$ We know also very little about the law, which guarantees the stability of the whole system of elementary particles interacting with each other and changing from one to another. Probably we need a broader background (such as the five-dimensional space or the quantized phase space) for field theory in order to cope with these problems, although it is premature to say anything definite in this connection. ${ }^{66}$

62 Hamilton, Heitler, and Peng, Phys. Rev. 64, 78 (1943) W. Heitler and P. Walsh, Rev. Mod. Phys. 17, 252 (1945) and many other papers on related subjects.

${ }_{63}$ Lewis, Oppenheimer, and Wouthysen, Phys. Rev. 73, 127 (1948).

${ }_{64}$ Morette and Peng, Nature 160, 59 (1947); Proc. Roy. Irish Acad. 51, 217 (1948); L. L. Foldy and R. E. Marshak, Phys. Rev. 75,1307 (1949). The author is indebted to Miss Morette for informing him of other unpublished results obtained by her.

${ }^{65}$ Very recently, Born and Green (Nature 163, 207, 208 (1949)) obtained a mass spectrum for elementary particles starting from the idea of self-reciprocity. Although their spectrum may not agree with the mass distribution realized in nature, it is very interesting that a mass spectrum was deduced purely field theoretically, probably for the first time.

${ }^{66}$ See, for example, H. Yukawa, Prog. Theor. Phys. 2, 209, (1947); 3, 205, 452 (1949); Phys. Rev. 76 (1949), in press. 